\title{
On the longitudinal component of paraxial fields
}

\author{
Artur Carnicer ${ }^{1}$, Ignasi Juvells ${ }^{1}$, David Maluenda ${ }^{1}$, Rosario \\ Martínez-Herrero ${ }^{2}$, and Pedro M. Mejías ${ }^{2}$ \\ ${ }^{1}$ Universitat de Barcelona (UB), Departament de Física Aplicada i Òptica, Facultat \\ de Física, Marti i Franquès 1, 08028 Barcelona (Spain) \\ ${ }^{2}$ Universidad Complutense de Madrid. Departamento de Óptica, Facultad de \\ Ciencias Físicas, Ciudad Universitaria, 28040 Madrid (Spain) \\ E-mail: artur.carnicer@ub.edu
}

\begin{abstract}
The analysis of paraxial Gaussian beams is a topic commonly present in undergraduate courses in Laser Physics, Advanced Optics, and Photonics. These beams provide a simple model of the field generated in resonant cavities of lasers, thus becoming a basic element for understanding the laser theory. Usually, uniformly polarized beams are considered in the analytical calculations, with the electric field vibrating at normal planes to the propagation direction. However, such paraxial fields do not verify the Maxwell equations. In this paper we discuss how to overcome this apparent contradiction and we evaluate the longitudinal component that any paraxial Gaussian beam should exhibit. Despite the fact that the assumption of a purely transverse paraxial field is useful and accurate, the inclusion of the above issue in the program helps students to clarify the importance of the electromagnetic nature of light, thus providing a more complete understanding of the paraxial approach.
\end{abstract}

PACS numbers: 01.40.-d, 41.20.Jb, 42.30.Kq, 42.60.Jf

Submitted to: Eur. J. Phys.

Version: 25 June 2012, 20:52

This paper is dedicated to the memory of our colleague and friend professor Pedro M. Mejías who recently died unexpectedly. 


\section{Introduction}

The study of the propagation of Gaussian beams (GB) is a common topic in most of the undergraduate courses in Lasers, Advanced Optics, and Photonics. In general, textbooks in these fields include a chapter covering this issue [1-6]. Furthermore, multiple papers highlighting the formative value of GB have been published: theoretical consideration, labs, simulations, etc [7-14]. At least two reasons justify the inclusion of GB in undergraduate programs: on the one hand, GB are solutions of the paraxial Helmholtz equation. The formula that describes the propagation of this kind of beams is easily derived and illustrates its physical behavior in an elegant and relatively simple way. On the other hand, nearly GB are generated in laser cavities, so they become a basic element for the understanding of laser theory.

In the analysis shown in the textbooks, a scalar approach is usually considered, which implicitly involves the assumption of a uniformly polarized field throughout the transverse wavefront. More precisely, the light beam is assumed to be uniformly linearly polarized, whose electric-field vector vibrates in transverse planes orthogonal to the direction of propagation.

However, strictly speaking, this type of paraxial fields does not verify the Maxwell equations since the divergence of such free-propagating beams is not zero. The failure of this law can be overcome just considering that the electric field is not confined to the transverse plane, i.e., its longitudinal component is not zero.

Nowadays, the study of light fields with significant longitudinal component is an active research topic either from the theoretical or from the technological point of view. Highlyfocused (non-paraxial) beams provide current examples of such fields. They are present, for instance, in high resolution optical microscopy, high density optical storage, or optical trapping, among other applications [15]. As is well known, non-paraxial beams have to be studied within the framework of the electromagnetic theory of diffraction $[16,17]$. Despite of the fact that it is not advisable to introduce this issue in undergraduate courses, the analysis of the longitudinal component in paraxial beams is affordable to these students. At the end of the day, the inclusion of this feature in the program provides a better understanding of the paraxial approach, and makes possible to clarify the link with a more advanced theory.

In this paper attention is mainly focused on how to estimate the longitudinal component of paraxial GBs. The article is organized as follows: In the next section we briefly review the formalism of the angular spectrum of plane waves. This simple and elegant formulation of propagation is equivalent to the conventional Sommerfeld scalar theory of diffraction [18]. In section 3, the properties of paraxial Gaussian beams are described [3]. In section 4, we show how to calculate the longitudinal component of a paraxial field at the convergence plane. For the particular but conceptually important case of GBs, results can be derived analytically. In section 5 we present an electromagnetic simulation to corroborate the previous calculations. The profile of the students to whom this material is intended is discussed in section 6. Finally, the main conclusions 
are summarized in section 7 .

\section{Beam propagation in terms of the angular spectrum of plane waves}

Let us first introduce some basic definitions and properties used in the paper. Let $\mathbf{E}(x, y, z)$ be an electromagnetic wave propagating along the direction $\mathbf{s}=(\alpha, \beta, \gamma)$; being $\mathbf{s}$ a unit vector, $|\mathbf{s}|=1$. For simplicity, the temporal dependence is omitted in the expressions. At $z=0$ the two-dimensional Fourier transform of $\mathbf{E}(x, y, 0)$ reads

$$
\hat{\mathbf{E}}(u, v, 0)=\operatorname{FT}[\mathbf{E}(x, y, 0)]=\int \mathbf{E}(x, y, 0) e^{-2 \pi i(u x+v y)} \mathrm{d} x \mathrm{~d} y,
$$

where $u$ and $v$ are the spatial frequencies and FT stands for the Fourier transform operator. Equivalently, $\mathbf{E}(x, y, 0)$ is the inverse Fourier transform of $\hat{\mathbf{E}}(u, v, 0)$ :

$$
\mathbf{E}(x, y, 0)=\mathrm{FT}^{-1}[\hat{\mathbf{E}}(u, v, 0)]=\int \hat{\mathbf{E}}(u, v, 0) e^{2 \pi i(u x+v y)} \mathrm{d} u \mathrm{~d} v .
$$

Since at $z=0$ a plane wave is described by $\mathbf{E}=\mathbf{A} e^{i k(\alpha x+\beta y)}$, being $\mathbf{A}$ the amplitude of the wave, $\lambda$ the wavelength and $k=2 \pi / \lambda$, Equation 2 can formally be understood as a superposition of plane waves traveling along a direction defined by the cosines $\alpha=\lambda u$, $\beta=\lambda v$ and $\gamma=\sqrt{1-\alpha^{2}-\beta^{2}}$, i.e.:

$$
\mathbf{E}(x, y, 0)=\int \hat{\mathbf{E}}(\alpha, \beta, 0) e^{i k(\alpha x+\beta y)} \mathrm{d} \frac{\alpha}{\lambda} \mathrm{d} \frac{\beta}{\lambda} .
$$

The contribution of each plane wave to the total field, namely $\hat{\mathbf{E}}(\alpha, \beta, 0)$, is the angular spectrum of plane waves associated to $\mathbf{E}(x, y, 0)$. In addition, the angular spectrum in any plane transverse to the longitudinal axis $z$ given by:

$$
\hat{\mathbf{E}}(\alpha, \beta, z)=\int \mathbf{E}(x, y, z) e^{-i k(\alpha x+\beta y)} \mathrm{d} x \mathrm{~d} y .
$$

Let us now write out the propagation of a beam in terms of its angular spectrum. By using the Helmholtz equation, $\nabla^{2} \mathbf{E}+k^{2} \mathbf{E}=0$, we get in the Fourier space

$$
\frac{\mathrm{d}^{2}}{\mathrm{~d} z^{2}} \hat{\mathbf{E}}(\alpha, \beta, z)+k^{2}\left(1-\alpha^{2}-\beta^{2}\right) \hat{\mathbf{E}}(\alpha, \beta, z)=0
$$

whose solution reads

$$
\hat{\mathbf{E}}(\alpha, \beta, z)=\hat{\mathbf{E}}(\alpha, \beta, 0) \exp \left(i k z \sqrt{1-\alpha^{2}-\beta^{2}}\right),
$$

being $H(\alpha, \beta, z)$ the so-called Transfer Function of the propagation in free space:

$$
H(\alpha, \beta, z)=\exp \left(i k z \sqrt{1-\alpha^{2}-\beta^{2}}\right) .
$$

Consequently, the propagated angular spectrum is obtained by calculating the product between the original angular spectrum and the transfer function. The scheme shown in Figure 1 summarizes the calculations. 


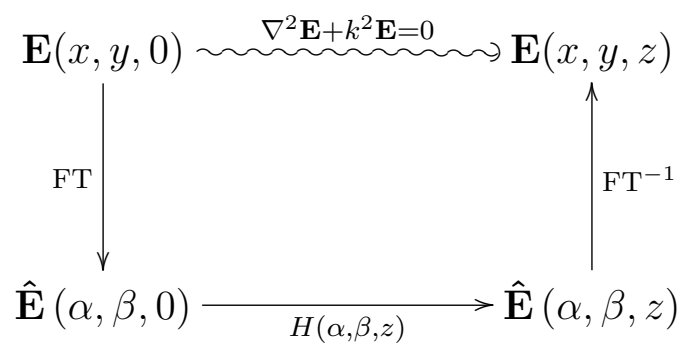

Figure 1: Calculation of $\mathbf{E}(x, y, z)$ using the propagation of the angular spectrum.

Up to now, we wrote no limits in the integrals of equations 1-4. However, in a rigorous way, the integration should be extended throughout the entire transverse plane. Consequently, two intervals should be considered: $\alpha^{2}+\beta^{2} \leq 1$ and $\alpha^{2}+\beta^{2}>1$. If the plane waves required to accurately describe the wavefront only take significant values close to the axis, i.e., when $\alpha^{2}+\beta^{2} \ll 1$ (paraxial or Fresnel approximation), the transfer function would be

$$
H_{F}(\alpha, \beta, z)=\exp (i k z) \exp \left(-i \frac{k z}{2}\left(\alpha^{2}+\beta^{2}\right)\right) .
$$

For these beams, the Helmholtz equation is reduced to

$$
\frac{\partial^{2}}{\partial x^{2}} \mathbf{A}(x, y, z)+\frac{\partial^{2}}{\partial y^{2}} \mathbf{A}(x, y, z)+2 i k \frac{\partial}{\partial z} \mathbf{A}(x, y, z)=0
$$

with $\mathbf{E}(x, y, z)=\mathbf{A}(x, y, z) e^{i k z}$. Note that for paraxial beams $\mathbf{A}(x, y, z)$ is a slowly variant function of $z$.

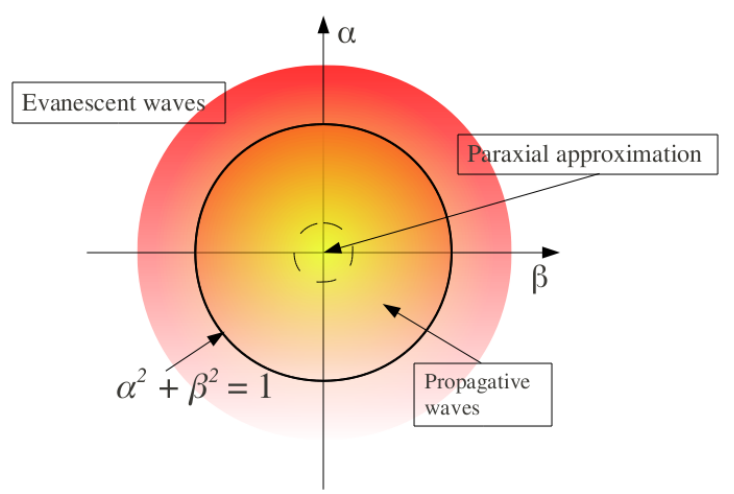

Figure 2: Propagative and evanescent regimes in the Fourier domain. The circle $\alpha^{2}+\beta^{2}=1$ indicates the limit of propagative and evanescent waves.

On the other hand, if the values of $\alpha$ and $\beta$ required to describe the beam are high enough, then $\alpha^{2}+\beta^{2}>1$. In this case the transfer function associated to this spatialfrequency interval becomes

$$
H_{E}(\alpha, \beta, z)=\exp \left(-k z \sqrt{\alpha^{2}+\beta^{2}-1}\right)
$$


and we see that the field (evanescent wave) vanishes very quickly following an exponential decay. Figure 2 displays the plane $(\alpha, \beta)$ showing the different possible calculation regimes. The interested reader can consult [18] for an extensive explanation about this topic.

\section{Paraxial Gaussian beams}

Gaussian beams propagating along the $z$-axis are paraxial waves (solutions of Equation 9) described by

$$
\begin{aligned}
& \mathbf{E}(x, y, z)=A_{G}(x, y, z) e^{i k z} \mathbf{p} \\
& A_{G}(x, y, z)=A_{0} \frac{w_{0}}{w(z)} \exp \left(-\frac{x^{2}+y^{2}}{w^{2}(z)}\right) \exp \left(i k \frac{x^{2}+y^{2}}{2 R^{2}(z)}\right) \exp (i \xi(z)),
\end{aligned}
$$

where $\mathbf{p}$ is a vector $(a, b, 0)$ that characterizes the polarization, $w(z)$ is the beam width radius, $w_{0}=w(0)$ is the waist radius, $R(z)$ is the radius of curvature, $z_{R}$ is the Rayleigh distance, $\xi(z)$ is the so-called Gouy phase, and $A_{0}$ is a constant. These parameters are given by the following expressions:

$$
\begin{aligned}
& w(z)=w_{0} \sqrt{1+\left(\frac{z}{z_{R}}\right)^{2}} \\
& \xi(z)=\arctan \left(\frac{z}{z_{R}}\right) \\
& R(z)=z\left(1+\left(\frac{z_{R}}{z}\right)^{2}\right) \\
& z_{R}=\frac{\pi w_{0}^{2}}{\lambda}
\end{aligned}
$$

and their geometrical interpretation is depicted in Figure 3. They provide meaningful information about how the Gaussian beam is propagated. The interpretation of these equations can be found elsewhere (see for instance [3]), but to aid understanding, the following information is provided:

- $\mathbf{p}$ is a vector $(a, b, 0)$ that characterizes the polarization, and $a$ and $b$ are the components of the Jones vector $(a, b)$. For example, for the linearly polarized Gaussian beam in the $x$-direction, $\mathbf{p}=(1,0,0)$ whereas for the circularly polarized Gaussian beam, $\mathbf{p}=\frac{1}{\sqrt{2}}\left(1, e^{i \pi / 2}, 0\right)$. Note that the third component of $\mathbf{p}$ is always zero for paraxial waves (i.e. the electric field vector vibrates in transverse planes orthogonal to the direction of propagation.)

- The divergence angle $2 \theta$ (see Figure 3 ) is defined as

$$
\theta=\arctan (w(z) / z) \approx \lambda /\left(\pi w_{0}\right) \quad\left(\text { for } z \gg z_{R}\right) .
$$

Equation 13 yields an interesting result: beams with large divergence angles focus in small spots and vice versa. Figure 4 a displays the variation of the beam waist 


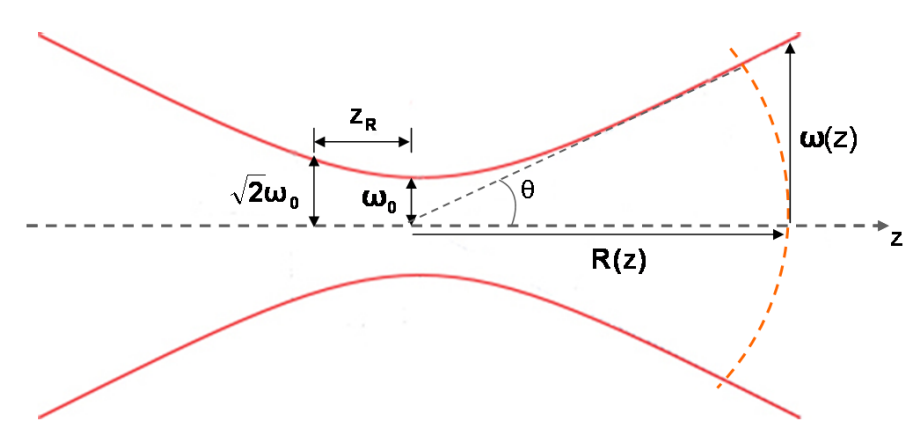

Figure 3: (a) Physical/geometrical interpretation of the beam parameters $w(z), R(z)$, $w_{0}, z_{R} . \theta$ is the divergence angle.

radius $w(z)$ as a function of the axial distance $z$. It is straightforward to show that for large values of $z$, the beam waist is proportional to the divergence angle: $w(z)=\theta z$.

- Figure $4 \mathrm{~b}$ shows the behavior of the irradiance along the optical $z$-axis

$$
|E(0,0, z)|^{2} \propto \frac{1}{1+\left(z / z_{R}\right)^{2}}
$$

for different values of the beam waist radius $w_{0}$. Note that the irradiance varies faster for small values of $w_{0}$, generating a narrow peak around the focus. In contrast, large values of $w_{0}$ produce a smoother variation of $|E(0,0, z)|^{2}$.

- At $z=z_{R}$ (the Rayleigh distance) the beam width is $w\left(z_{R}\right)=\sqrt{2} w_{0}$ and the irradiance drops to $\left|E\left(0,0, z_{R}\right)\right|^{2}=0.5$. The confocal parameter (or depth of focus) is defined as the range of values $z$ that ensure $z \in\left[-z_{R}, z_{R}\right]$. Focused beams present a small depth of focus.

- The wavefront of the Gaussian beam tends to be spherical for large values of $z$ since the radius of curvature becomes $R(z) \approx z$ for $z \gg z_{R}$. At $z=0, R(z) \rightarrow \infty$ and thus the wavefront is plane at the focus.

- The Gouy phase introduces a phase shift as the beam propagates, with $\pi$ being the total delay for a beam propagating from $-\infty$ to $\infty$. Moreover, notice that the sign of the Gouy phase changes at $z=0$.

- Figures 5a and 5b map the distribution $|E(x, 0, z)|^{2}$ for $w_{0}=2 \lambda$ and $w_{0}=5 \lambda$ respectively. These figures are displayed in false color using the Matlab default jet colormap and normalized to the maximum value (small values are displayed in blue whereas large values are shown in red). These figures help emphasize some important issues in the analysis: small values of $w_{0}$ produces beams with high divergence angles and small depth of focus, and vice versa. 
Finally, it is worth to mention here that other beams can be solutions to the paraxial Helmholtz equation (Equation 9). In particular, the so called Hermite-Gauss beams are complete set of solutions whose irradiance is given by

$$
\left|E_{m n}(x, y, z)\right|^{2} \propto\left|H_{m}\left(\frac{\sqrt{2} x}{w(z)}\right) H_{n}\left(\frac{\sqrt{2} y}{w(z)}\right) E(x, y, z)\right|^{2},
$$

being $H_{n}(x)$ the Hermite polynomial of order $n$; for instance, $H_{0}(x)=1$ and $H_{1}(x)=2 x$. Since $H_{0}(x)=1$, the Hermite-Gauss beam of order $(0,0)$ is just the paraxial Gaussian beam.

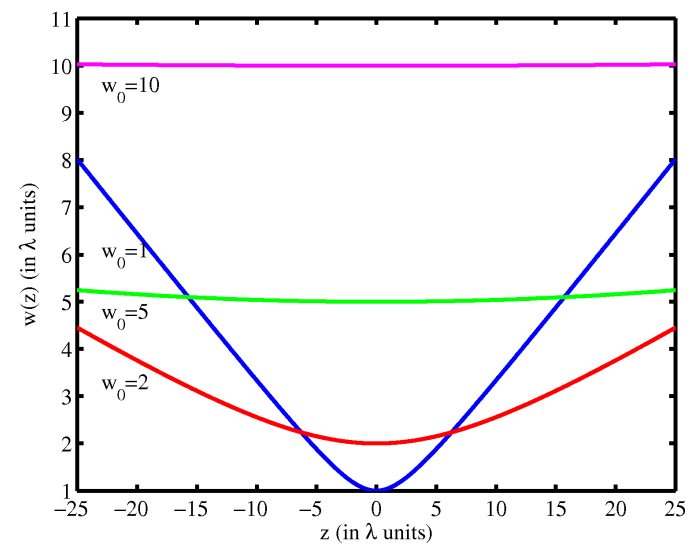

(a)

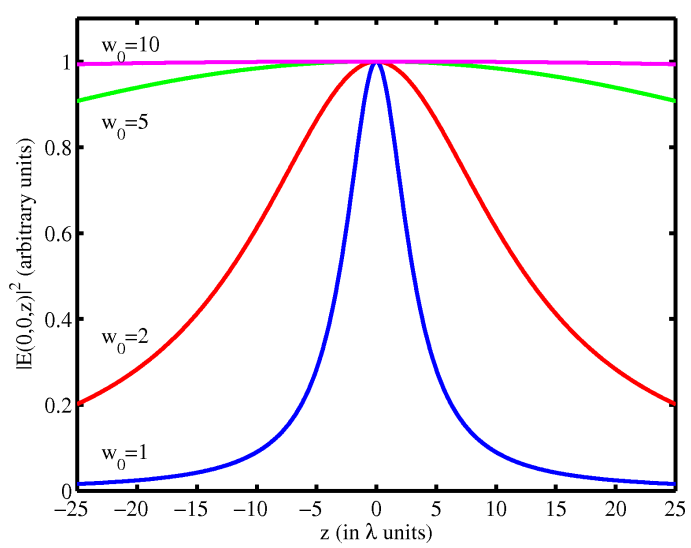

(b)

Figure 4: (a) Dependence of the beam radius $w(z)$ and (b) the irradiance $|E(0,0, z)|^{2}$ along the optical axis for different values of $w_{0}$.

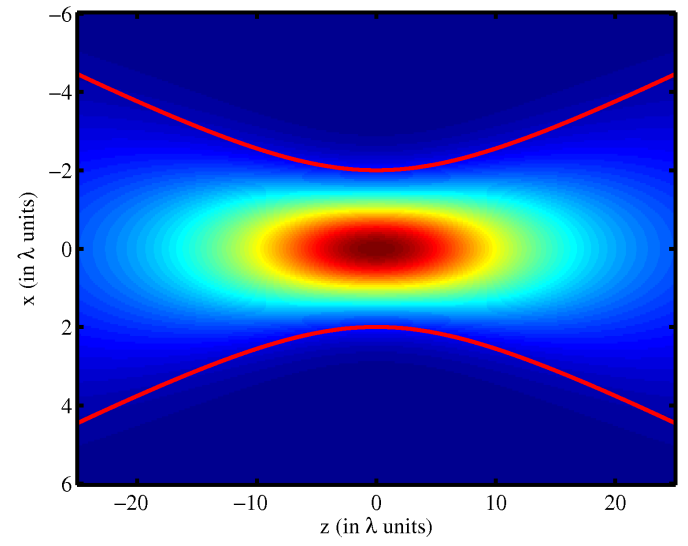

(a)

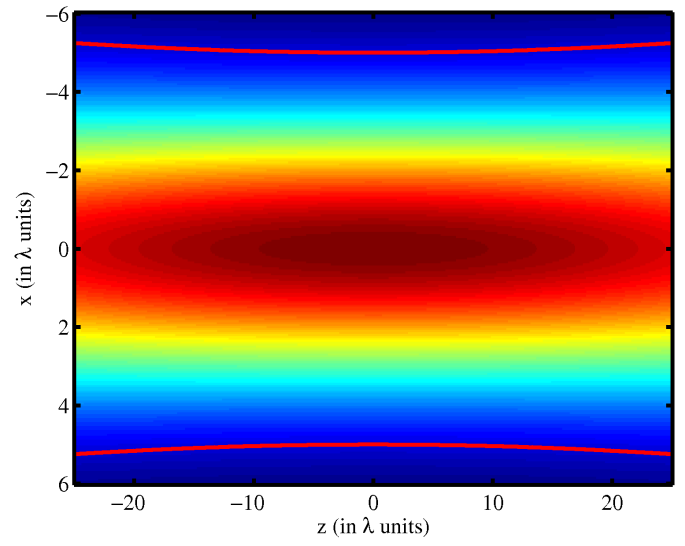

(b)

Figure 5: Map of the irradiance $|E(x, 0, z)|^{2}$ for (a) $w_{0}=2 \lambda$ and (b) $w_{0}=5 \lambda$. The red line superimposed to each image represents the beam width. 


\section{Longitudinal component of paraxial Gaussian beams}

An electromagnetic beam propagating in a medium with no charges nor currents must fulfill the divergence condition (Gauss' Law) $\nabla \mathbf{E}=0$. Nevertheless, it is straightforward to show that the paraxial Gaussian beam does not verify Gauss' Law, since the uniformly-polarized electric field vibrates in the transverse plane, and the $z$-component is always zero $\ddagger$. To avoid this apparent contradiction, let us consider a paraxial beam with non-zero longitudinal component $E_{z}$, which is evaluated by using the Gauss' Law:

$$
\frac{\partial E_{z}}{\partial z}=-\frac{\partial E_{x}}{\partial x}-\frac{\partial E_{y}}{\partial y}
$$

In terms of its angular spectrum, $\hat{\mathbf{E}}=\left(\hat{E}_{\alpha}, \hat{E}_{\beta}, \hat{E}_{z}\right)$ (equation 4), the Gauss' Law is written $\mathbf{s} \hat{\mathbf{E}}=0$, thus Equation 16 becomes

$$
\hat{E}_{z}=-\frac{\alpha \hat{E}_{\alpha}+\beta \hat{E}_{\beta}}{\sqrt{1-\alpha^{2}-\beta^{2}}},
$$

and we obtain the longitudinal component $E_{z}$ from the inverse Fourier transform, i.e., $E_{z}=\mathrm{FT}^{-1}\left[\hat{E}_{z}\right]$. A linearly polarized Gaussian field in the $x$-direction with $w_{0}=10 \lambda$ has been evaluated numerically. Figure 6 displays $\left|E_{x}\right|^{2}$ and $\left|E_{z}\right|^{2}$ at the plane $z=0$. The images are presented again in false color using the jet colormap and normalized to its maximum value.

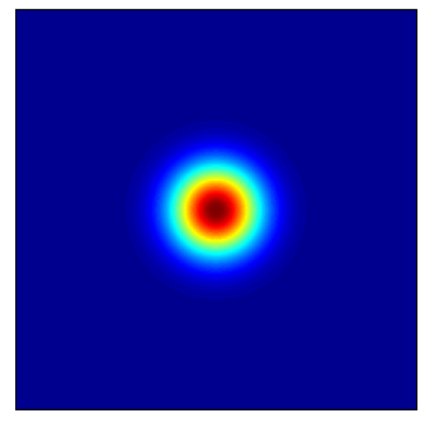

(a) $\left|E_{x}\right|^{2}$

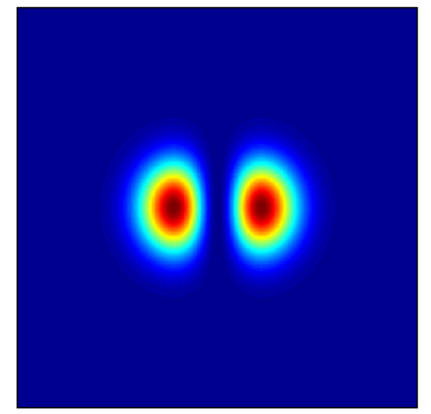

(b) $\left|E_{z}\right|^{2}$

Figure 6: Squared modulus of the field components $E_{x}$ and $E_{z}$ at $z=0$. The paraxial Gaussian beam is assumed to be linearly polarized throughout the transverse plane. The length of the side of the images is $L=64 \lambda$.

$\ddagger$ It is worth pointing out that purely transverse beams (i.e., without longitudinal component) can propagate satisfying the Maxwell equations. A certain type of azimuthally polarized fields constitutes an illustrative example of such beams [19]. But no contradiction exists with the present paper because azimuthally polarized fields are not uniformly polarized at a transverse plane, as is assumed throughout this work. 


\begin{tabular}{|l|l|l|l|}
\hline & $I_{x} / I_{T}$ & $I_{y} / I_{T}$ & $I_{z} / I_{T}$ \\
\hline Linearly polarized case & 0.99974 & 0 & 0.00026 \\
\hline Circularly polarized case & 0.49987 & 0.49987 & 0.00026 \\
\hline
\end{tabular}

Table 1: Integrated irradiance for each component of the electric field. Results are normalized to $I_{T}=I_{x}+I_{y}+I_{z}$.

Furthermore, the irradiance associated to the longitudinal and transversal components throughout the plane $z=0$ is proportional to the following integrals:

$$
\begin{aligned}
& I_{x}=\int\left|E_{x}\right|^{2} \mathrm{~d} x \mathrm{~d} y \\
& I_{z}=\int\left|E_{z}\right|^{2} \mathrm{~d} x \mathrm{~d} y .
\end{aligned}
$$

In the linearly polarized case in the $x$-direction, $I_{z}$ is about $10^{-4}$ times smaller than $I_{x}$, (see Table 1). For the circularly polarized case, the results are shown in Figure 7 and Table 1 as well. Notice that the ratio $I_{z} / I_{T}$ takes the same value in both cases.

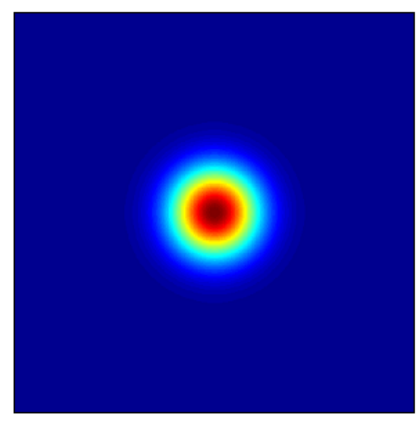

(a) $\left|E_{x}\right|^{2}$

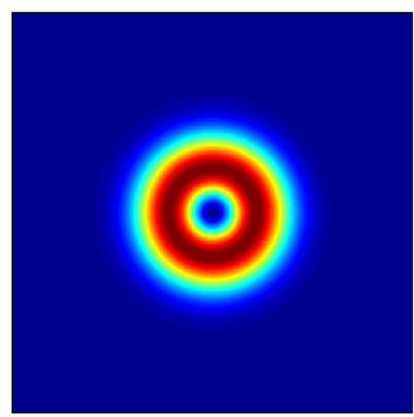

(b) $\left|E_{z}\right|^{2}$

Figure 7: The same as in Figure 6 but now computed for the circularly-polarized case. As should be expected, $\left|E_{y}\right|^{2}$ is identical to $\left|E_{x}\right|^{2}$.

Figure 8 shows the ratio $I_{z} / I_{T}$ at $z=0$ as a function of the waist size. We see that $I_{z}$ increases for small values of the beams width $w_{0}$. But smaller $w_{0}$ means higher divergence (cf. equations 12). Thus we conclude that for nearly collimated beams, the longitudinal component becomes negligible compared with the transverse ones. On the contrary, the type of highly focused fields we are considering would exhibit a significant longitudinal component. Of course, strictly speaking, the longitudinal component is never zero. 


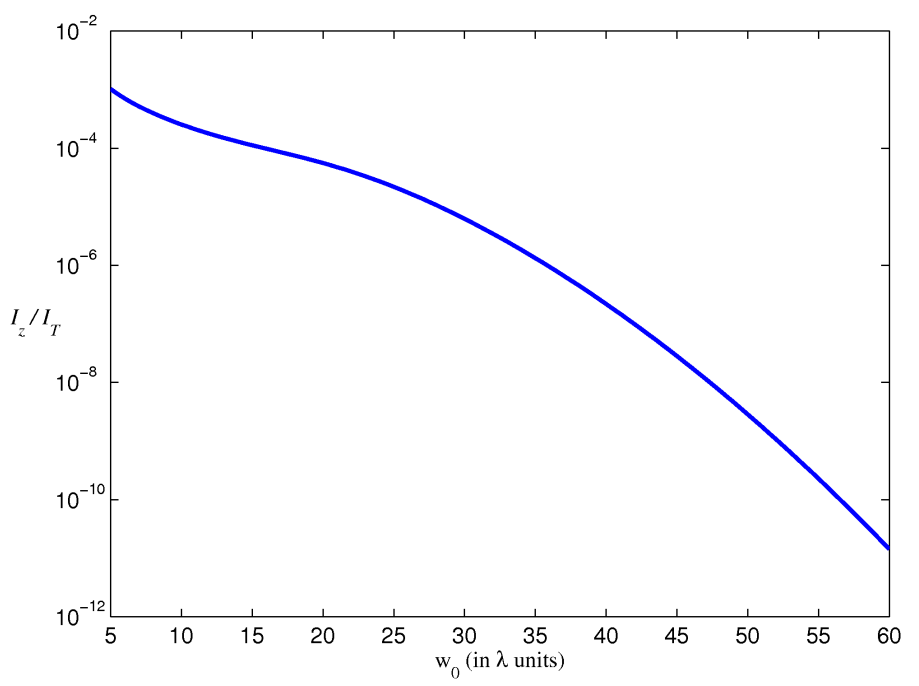

Figure 8: Ratio $I_{z} / I_{T}$ as a function of the beam waist $w_{0}$.

\section{An analytical derivation}

The previous calculations were carried out numerically. This procedure is valid for any paraxial beam. Nevertheless, it is possible to derive analytical expressions for the longitudinal component of Gaussian beams [15]. Let us consider first the linearly polarized case:

$$
\mathbf{E}(x, y, 0)=A_{0} \exp \left(-\frac{x^{2}+y^{2}}{w_{0}^{2}}\right)(1,0,0) .
$$

Taking into account that, for paraxial beams $\alpha \ll 1, \beta \ll 1$ and $\gamma \approx 1$, Equation 17 becomes

$$
\hat{E}_{z} \approx-\alpha \hat{E}_{\alpha}=-\frac{\lambda}{2 \pi i} 2 \pi i u \hat{E}_{\alpha}
$$

Using the derivative property of Fourier transforms, namely,

$$
\mathrm{FT}\left[\frac{\partial E_{x}}{\partial x}\right]=2 \pi i u \mathrm{FT}\left[E_{x}\right]=2 \pi i u \hat{E}_{\alpha}
$$

the longitudinal component at $z=0$ would read

$$
E_{z}(x, y, 0) \approx-\frac{\lambda}{2 \pi i} \frac{\partial}{\partial x} E_{x}(x, y, 0)=\frac{A_{0}}{i k} \frac{2 x}{w_{0}^{2}} \exp \left(-\frac{x^{2}+y^{2}}{w_{0}^{2}}\right)
$$

where

$$
\left|E_{z}(x, y, 0)\right|^{2} \propto \frac{x^{2}}{w_{0}^{4}} \exp \left(-\frac{x^{2}+y^{2}}{w_{0}^{2}}\right)
$$

is proportional to the irradiance. From the previous Equation (see also Figure $6 \mathrm{~b}$ and Equation 15), we conclude that the longitudinal component at plane $z=0$ exhibit the 
same profile as the $(1,0)$ Hermite-Gauss mode. In a similar way, an analytical expression for the logitudinal component of a circularly-polarized Gaussian beam can be derived:

$$
\begin{aligned}
E_{z}(x, y, 0) & =-\frac{\lambda}{2 \pi i}\left(\frac{\partial}{\partial x}+\frac{\partial}{\partial y}\right) E_{x}(x, y, 0)= \\
& =\frac{A_{0}}{i k} \frac{2}{w_{0}^{2}}(x+i y) \exp \left(-\frac{x^{2}+y^{2}}{w_{0}^{2}}\right),
\end{aligned}
$$

and the irradiance becomes

$$
\left|E_{z}(x, y, 0)\right|^{2} \propto \frac{x^{2}+y^{2}}{w_{0}^{4}} \exp \left(-\frac{x^{2}+y^{2}}{w_{0}^{2}}\right) .
$$

Now, $\left|E_{z}\right|^{2}$ shows a doughnut shape, which can be understood as a superposition of the $(1,0)$ and $(0,1)$ Hermite-Gauss modes (in agreement with Figure $7 \mathrm{~b})$. Moreover, the $z$-component of the electric field (Equations 22 and 24) exhibits a $\frac{1}{w_{0}^{2}}$ dependence with the waist radius, thus $E_{z}$ becomes larger for smaller values of $w_{0}$.

\section{A more in depth analysis}

In the present work we used the conventional scalar diffraction theory to evaluate the longitudinal component of the electric field of a uniformly-polarized paraxial Gaussian beam. Regarding the linearly polarized case, we considered that the electric field vector at the initial plane has only the $x$-component, with the $y$-component equal to zero. However, a more in depth analysis based on the Electromagnetic Theory of Diffraction (ETD) shows an interesting non-null behavior of the $y$-component upon propagation. Moreover, the application of ETD provides the required theoretical foundation for the rigorous description of light propagation in the nano-scale. Despite the fact that it is not advisable to introduce ETD in undergraduate courses, a relatively simple calculation can provide us more insight about the inner structure of the three components of the electric field.

We have performed a simulation of the propagation of a $(x$-axis) linearly polarized Gaussian beam by using the finite-difference time-domain (FDTD) method [20]. FDTD tools are designed to integrate Maxwell's equations and are very useful to model electromagnetic systems. The calculation has been carried out using the MEEP $\mathrm{C}++$ library [21] §. A linearly-polarized converging Gaussian beam with $w_{0}=10 \lambda$ has been calculated at $z=-6.5 \lambda$ using equations 11 and 12 . The beam is propagated using MEEP until the waist plane $(z=0)$; to integrate across time, 840 frames during 50 periods have been considered. Figures 9a and 9c show the profiles $\left|E_{x}(x, y, 0)\right|^{2}$ and $\left|E_{z}(x, y, 0)\right|^{2}$. Both are indistinguishable with the corresponding distributions obtained analytically (cf. figures $6 \mathrm{a}$ and $6 \mathrm{~b}$ ). Moreover, the values $I_{x} / I_{T}$ and $I_{z} / I_{T}$ are very close to the values obtained in the previous section. It should be remarked that, $\left|E_{y}(x, y, 0)\right|^{2}$ (figure $9 \mathrm{~b}$ ) is not zero and looks as the $(1,1)$ Hermite-Gauss mode. Nevertheless, the 
value of the ratio $I_{y} / I_{T}$ is very low in front of $I_{x} / I_{T}$ or even $I_{z} / I_{T}$. These results are in agreement with previous works reported in the literature (see, for example, $[19,22]$ ).

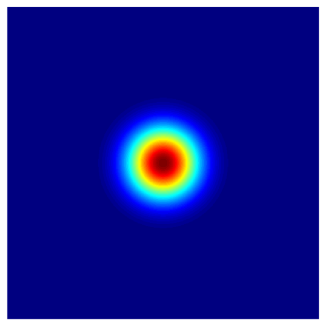

(a) $\left|E_{x}\right|^{2}$

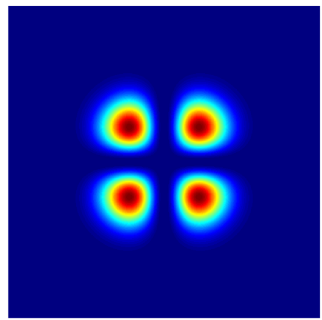

(b) $\left|E_{y}\right|^{2}$

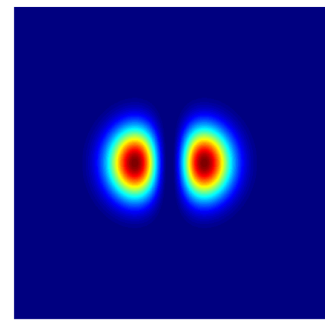

(c) $\left|E_{z}\right|^{2}$

Figure 9: Electric field components of the linearly polarized case, with $w_{0}=10 \lambda$. The length of side of the images is $\mathrm{L}=64 \lambda$. We have $I_{x} / I_{T}=0.9997, I_{y} / I_{T}=6.9010^{-8}$ and $I_{z} / I_{T}=2.9710^{-4}$.

For the sake of completeness, the propagation of a circularly polarized Gaussian beam using MEEP has also been considered. The simulation has been carried out using the same values as in the previous calculation. It is quite apparent that figures $9 \mathrm{a}$ and $9 \mathrm{c}$ are in complete agreement with figures $7 \mathrm{a}$ and $7 \mathrm{~b}$ obtained from analytical expressions.

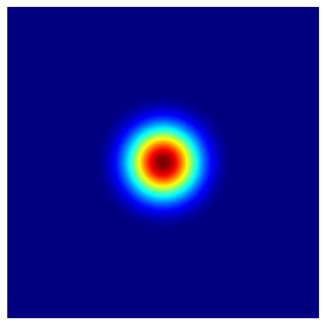

(a) $\left|E_{x}\right|^{2}$

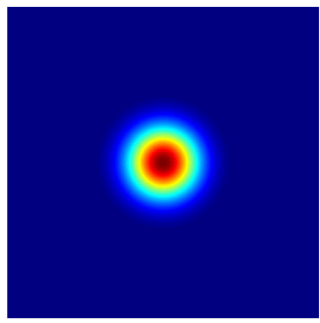

(b) $\left|E_{y}\right|^{2}$

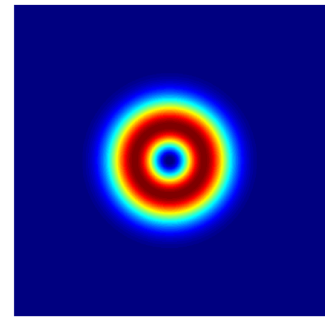

(c) $\left|E_{z}\right|^{2}$

Figure 10: The same as Figure 9 but for a circularly polarized beam. Now $I_{x} / I_{T}=$ $0,49985, I_{y} / I_{T}=0,49985$ and $I_{z} / I_{T}=2.9710^{-4}$ (the same value as in Figure 9).

\section{How this material relates to students}

The content of this paper was proposed for two different groups of students. On the one hand, this analysis was suggested as a discussion topic for students enrolled in Photonics, a compulsory subject for advanced undergraduates in Applied Physics at the Universitat de Barcelona. It comprises 60 hours of lectures and 9 labs: equivalent to 9 ECTS credits. Those who enroll on the course have previously taken compulsory credits in physical optics (which includes diffraction and beam propagation theory). 
The Photonics syllabus includes a relatively in-depth introduction to lasers, with two lectures devoted to the propagation of Gaussian beams. On average, about 25 students take Photonics each year and the pass rate is approximately $80 \%$.

On the other hand, a numerical verification of this work was carried out by graduate students taking a computational techniques course. Computing in Photonics is a 2.5 ECTS credit core subject that forms part of the Barcelona Master in Photonics: a oneyear graduate program offered jointly by three universities in the Barcelona metropolitan area. It comprises 20 hours of lectures and the homework workload is estimated at 45 hours. The syllabus includes Fourier transform-based beam propagation methods, finitedifference time domain methods and integration of differential equations. On average, about 15 students take the course each year and the pass rate is close to $90 \%$.

\section{Concluding remarks}

The study of light beams with Gaussian profile is present in undergraduate courses in physics. This kind of beams provides a simple solution of the paraxial Helmholtz equation and can be used for illustrating a number of physically meaningful spatial characteristics of paraxial light fields. Usually, for the sake of simplicity, uniformly polarized beams are handled in the analytical calculations, with the electric field vibrating in planes normal to the direction of propagation. However, a problem arises because this type of fields do not fulfill the divergence condition $\nabla \mathbf{E}=0$. This apparent contradiction is solved by noting that such fields exhibit a nonzero contribution in the longitudinal direction. Simple analytical expressions have been derived for the longitudinal component of a Gaussian beam. Two representative cases have been considered: linearly and circularly uniformly-polarized fields. It has been found that the squared modulus of this component resembles the spatial shape of certain HermiteGauss functions. Moreover, numerical computations have also been shown that strongly focused beams (in the non-paraxial regime) exhibit significant values of the magnitude of the longitudinal component, whereas its contribution is negligible (compared with the transverse components) for slowly converging beams. The results obtained have been verified by simulating free propagation of a Gaussian beam using a FDTD routine. In summary, the discussion on the presence of a longitudinal component in paraxial beams is an illustrative topic that provides a better understanding of the paraxial approximation for vectorial light fields. This topic is suitable for both advanced undergraduates and graduate students in Physics enrolled in Laser Theory, Fourier Optics or Photonics courses.

\section{References}

[1] A.E. Siegman. Lasers. University Science Books, 1986.

[2] W.T. Silfvast. Laser fundamentals. Cambridge University Press, 2004.

[3] B.E.A. Saleh and M.C. Teich. Fundamentals of photonics. John Wiley \& Sons, 2007. 
[4] D. Meschede. Optics, light and lasers: the practical approach to modern aspects of photonics and laser physics. Wiley-VCH, 2007.

[5] O. Svelto. Principles of lasers. Springer-Verlag, 2009.

[6] F.L. Pedrotti, L.S. Pedrotti, and L.M. Pedrotti. Introduction to optics. Prentice-Hall Englewood Cliffs, New Jersey, 1987.

[7] S.A. Boothroyd, L. Chan, and WM Robertson. Visualizing coherent light with an electromagnetic wave simulator. IEEE Transactions on Education, 39(1):29-39, 1996.

[8] AM Almeida, E. Nogueira, and M. Belsley. Paraxial imaging: Gaussian beams versus paraxialspherical waves. American Journal of Physics, 67:428, 1999.

[9] M. Jackson, D. Bauen, and JE Hasbun. Investigation of laser fundamentals using a helium-neon laser. European Journal of Physics, 22:211, 2001.

[10] PP Crooker, WB Colson, and J. Blau. Representation of a gaussian beam by rays. American Journal of Physics, 74:722, 2006.

[11] E.J. Galvez. Gaussian beams in the optics course. American Journal of Physics, 74:355, 2006.

[12] F.W. Sheu and C.H. Chang. Measurement of the intensity profile of a gaussian laser beam near its focus using an optical fiber. American Journal of Physics, 75:956, 2007.

[13] J. Mas, A. Farre, J. Cuadros, I. Juvells, and A. Carnicer. Understanding optical trapping phenomena: A simulation for undergraduates. IEEE Transactions on Education, (45):133-140, 2011.

[14] J. Henningsen. Teaching laser physics by experiments. American Journal of Physics, 79:85, 2011.

[15] L. Novotny and B. Hecht. Principles of nano-optics. Cambridge University Press, 2006.

[16] E. Wolf. Electromagnetic diffraction in optical systems. I. An integral representation of the image field. Proceedings of the Royal Society of London. Series A. Mathematical and Physical Sciences, 253(1274):349, 1959.

[17] P. Varga and P. Török. The gaussian wave solution of Maxwell's equations and the validity of scalar wave approximation. Optics Communications, 152(1-3):108-118, 1998.

[18] J.W. Goodman. Introduction to Fourier optics. Roberts \& Company Publishers, 2005.

[19] R. Martínez-Herrero, P.M. Mejías, and G. Piquero. Characterization of partially polarized light fields. Springer Verlag, 2009.

[20] A. Taflove and S.C. Hagness. Computational electrodynamics: the finite-difference time-domain method. Artech House Boston, 2005.

[21] A. F. Oskooi, D. Roundy, M. Ibanescu, P. Bermel, J. D. Joannopoulos, and S. G. Johnson. MEEP: A flexible free-software package for electromagnetic simulations by the FDTD method. Computer Physics Communications, 181:687-702, 2010.

[22] PM Mejías, R. Martínez-Herrero, G. Piquero, and JM Movilla. Parametric characterization of the spatial structure of non-uniformly polarized laser beams. Progress in Quantum electronics, 26(2):65-130, 2002.

\section{Acknowledgments}

This paper has been supported by the Ministerio de Ciencia e Innovación of Spain, project FIS2010-17543 and the Program for Teaching Improvement and Innovation (PMID) of the Universitat de Barcelona, project GIDCUB-11/OFF. 